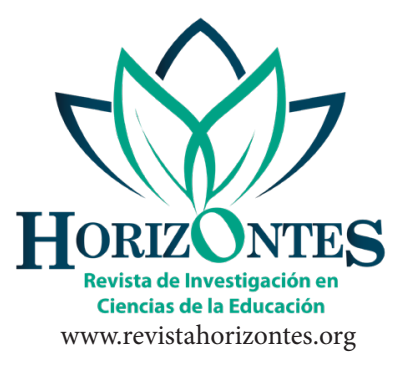

\title{
El desarrollo psicomotor y el aprendizaje de la iniciación de la lectoescritura en el nivel inicial
}

\author{
The psychomotor development and the learning of the initiation of the literacy in the initial level \\ O desenvolvimento psicomotor e a aprendizagem da iniciação à alfabetização no nível inicial
}

Claudia Katherine Gonzales Remigio

kty.clau@gmail.com

https://orcid.org/0000-0003-2074-8457

Universidad César Vallejo, Lima-Perú

Artículo recibido 24 de octubre 2021, arbitrado y aceptado 29 de noviembre 2021 y publicado 28 de enero 2022

\section{RESUMEN}

El estudio tuvo como objetivo la determinación de la relación entre el desarrollo psicomotor con el aprendizaje a la iniciación de la lectoescritura en una institución educativa de Lima. El estudio es de tipo básico y nivel descriptivo correlacional. El diseño no experimental de corte transversal. La población de estudio estuvo conformada por 120 estudiantes de 5 años, la muestra fue de 90 estudiantes. Se utilizó como instrumento el test TEPSI y el test BENHALE; el test correspondiente a la variable desarrollo psicomotor, comprende 52 ítems los cuales estuvieron divididos en tres según sus dimensiones motricidad, lenguaje y coordinación con 12,24 y 16 ítems respectivamente. Respecto a la variable aprendizaje a la lectoescritura el test BENHALE estuvo conformado por 80 ítems. Según los resultados obtenidos se tuvo que existe una relación significativa entre el nivel de desarrollo psicomotor y el aprendizaje a la iniciación de la lectoescritura en niños de cinco años de una institución educativa de Lima.

Palabras clave: Motricidad; Coordinación; Habilidades motoras; Desarrollo cognitivo
ABSTRACT

The objective of the study was to determine the relationship between psychomotor development and learning at the beginning of literacy in an educational institution in Lima. The study is of a basic type and descriptive correlational level. The nonexperimental cross-sectional design. The study population consisted of 1205 -yearold students; the sample was 90 students. The TEPSI test and the BENHALE test were used as instruments; The test corresponding to the psychomotor development variable comprises 52 items which were divided into three according to their motor, language and coordination dimensions with 12,24 and 16 items respectively. Regarding the variable learning to read and write, the BENHALE test consisted of 80 items. According to the results obtained, there was a significant relationship between the level of psychomotor development and learning at the beginning of literacy in five-year-old children from an educational institution in Lima.

Key words: Motor skills, Coordination; Motor skills; Cognitive development

\section{RESUMO}

O objetivo do estudo foi verificar a relação entre o desenvolvimento psicomotor e a aprendizagem no início da alfabetização em uma instituição de ensino de Lima. O estudo é de tipo básico e nível descritivo correlacional. O desenho transversal não experimental. A população do estudo foi composta por 120 alunos de 5 anos de idade, a amostra foi de 90 alunos. $\mathrm{O}$ teste TEPSI e o teste BENHALE foram utilizados como instrumentos; O teste correspondente à variável desenvolvimento psicomotor é composto por 52 itens que foram divididos em três de acordo com suas dimensões motora, de linguagem e coordenação com 12, 24 e 16 itens respectivamente. Em relação à variável aprender a ler e escrever, o teste BENHALE foi composto por 80 itens. De acordo com os resultados obtidos, houve relação significativa entre o nível de desenvolvimento psicomotor e a aprendizagem no início da alfabetização em crianças de cinco anos de uma instituição de ensino de Lima.

Palavras-chave: Habilidades motoras; Coordenação; Habilidades motoras; Desenvolvimento cognitivo 


\section{INTRODUCCIÓN}

Es importante que los individuos desarrollen su psicomotricidad, ya que esto permite que estén en contacto con el ambiente que les rodea, potenciando el proceso de enseñanza y aprendizaje y permitiendo el desarrollo de los infantes. Es por esto que en la etapa escolar los niños aprenden a través de actividades motrices, esto les permite la interacción con su medio; por esta razón la psicomotricidad es considerada una herramienta que ayuda al estudiante a tomar conciencia del tiempo, espacio y de su cuerpo (Hernández, 2021).

Los infantes se expresan a través de su cuerpo, de llantos, movimientos, entre otros; esto forma parte de un proceso de comunicación frente a situaciones que muchas veces no se pueden comunicar a través de palabras, convirtiéndose también en un medio por el que descargan sus tensiones y exploran el mundo. En ese sentido, la educación corporal es uno de los puntos de acción en las escuelas, ya que provee una serie de experiencias que facilitan el desarrollo de las capacidades. Sin embargo, es necesario tener en cuenta que este desarrollo está condicionado a muchos aspectos, como los factores sociales, culturales, escolares y familiares (Roberto, 2018).

La madurez que tienen los niños es importante cuando ingresan al sistema escolar, porque esto les permite enfrentarse a las exigencias escolares, ya que cuando están aún pequeños se enfrentan principalmente al aprendizaje de iniciación a la lectoescritura, el cual representa un proceso complejo para ellos (Saavedra y Tello, 2015). Respecto al desarrollo psicomotor y la iniciación a la lectoescritura, el desarrollo psicomotor juega un papel importante ya que interviene en los procesos madurativos del infante e influye en la adquisición de habilidades básicas las cuales permiten el aprendizaje de la escritura, siendo que esta habilidad abarca un conjunto de habilidades motoras cognitivas, perceptivas y visuales (Espinoza et al., 2017).

Según la Organización de las Naciones Unidas para la Educación, la Ciencia y la Cultura (2017) son más de 617 millones los adolescentes y niños que no han alcanzado los niveles de aprendizaje que se requieren en las áreas de matemáticas y lectura, esto refleja una gran disminución en el potencial humano y un retraso hacia el cumplimiento de los Objetivos de Desarrollo Sostenible. Asimismo, los resultados del examen internacional PISA, evidenciaron que la mayoría de países presentan dificultades respecto a la comprensión lectora, observándose que el $20 \%$ de los estudiantes que participaron en este examen, no superan el promedio fijado por la OCDE, esto debido a que no cuentan con métodos adecuados que les facilite la comprensión de lo que leen. Estas evaluaciones destacan las carencias que existen en el sistema educativo (OCDE, 2017).

Respecto a la iniciación a la lectoescritura en Latinoamérica y el Caribe, Stone (2019) evidenció que para mejorar los resultados en la lectura es importante tener en cuenta el contexto, ya que esto permite un mejor enfoque en los programas y estrategias, considerando las diferencias culturales, económicas y políticas de cada país. Asimismo, a nivel general resalta que para Latinoamérica el trabajo infantil y la pobreza, tienen una correlación negativa con los resultados esperados en la lectoescritura inicial. Además, destaca que una educación de calidad en pre escolar está asociada positivamente con la lectoescritura inicial. 
La problemática local refiere que existen muchos niños los cuales no tienen la madurez suficiente, ya que no han desarrollado las funciones básicas para el aprendizaje, esto genera frustración, fracaso, deserción escolar, entre otros. Es así que es indispensable la identificación temprana de los estudiantes que tienen algún riesgo social o biológico a través de test que permitan predecir la madurez en el proceso de aprendizaje de la lectoescritura en los niños y la dificultades que estos presentan (Saavedra y Tello, 2015).

Es así que tomando en cuenta lo descrito en párrafos anteriores, se plantea la siguiente pregunta general: ¿De qué manera se relaciona el desarrollo psicomotor y el aprendizaje a la iniciación de la lectoescritura en los niños de 5 años de la una institución educativa en Lima?, por otro lado, las preguntas específicas tomaron en consideración ${ }_{i}$ De qué manera se relaciona el desarrollo psicomotor en su modalidad lenguaje, coordinación y motricidad con el aprendizaje de la lectoescritura en niños de 5 años de la Institución Educativa de Lima?

La investigación tuvo como objetivo conocer la relación entre el desarrollo psicomotor y el aprendizaje a la iniciación de la lectoescritura en niños de 5 años de una institución educativa en Lima, además los objetivos específicos plantean conocer la relación del desarrollo psicomotor en su modalidad lenguaje, coordinación y motricidad, con el aprendizaje a la iniciación de la lectoescritura en niños de 5 años de una institución educativa en Lima.

La hipótesis general del estudio plantea que existe una relación significativa entre desarrollo psicomotor y el aprendizaje a la iniciación de la lectoescritura en niños de 5 años de una institución educativa en Lima, asimismo las hipótesis específicas plantean que existe relación significativa entre el lenguaje, coordinación y motricidad con el aprendizaje a la Iniciación de la lectoescritura en niños de 5 años de una institución educativa en Lima.

La justificación teórica de la investigación radica en que se presenta información actualizada y especializada relacionada al desarrollo psicomotor del infante, el cual influye en el lenguaje, coordinación y motricidad. Su justificación social radica en que el grupo etéreo abarca niños de inicial de 5 años.

Respecto a los fundamentos teóricos relacionados con la variable desarrollo psicomotor Arteaga et al. (2001) lo definen como la adquisición progresiva de habilidades sociales, biológicas y psicológicas en el infante, esto implica cambios progresivos, secuenciales e irreversibles en el individuo en desarrollo. Comprende tres aspectos estos son la motricidad, lenguaje y coordinación. Asimismo, Doussoulin (2003) lo define como el desarrollo multidimensional, en el que se realizan cambios continuos, donde el infante se desenvuelve en niveles cada vez más complejos involucrando cambios en el plano físico o motor, intelectual y sensorial, siendo estos planos de desarrollo indispensables para el correcto crecimiento y maduración del niño. Por otro lado, Rosso (2008) lo define como un proceso en el que el niño puede conocer, relacionarse y adaptarse al contexto que lo rodea, involucrando factores como el lenguaje comprensivo y expresivo la motricidad el equilibrio coordinación visomotora y el aspecto social afectivo.

Respecto a la variable lectoescritura Diez (1999) lo menciona como un proceso interactivo 
donde el niño juega un papel activo en el que tiene la capacidad de reflexionar y estar en busca del significado de sus pensamientos e ideas. El autor enfatiza en la necesidad que tienen los niños en pensar acerca de lo que escriben y leen y es importante tener en cuenta el trabajo de los docentes y facilitadores que ayudan a la comprensión de la realidad del niño. El autor agrega qué el aprendizaje de la lectoescritura no se puede realizar de manera individual, ya que es importante la interacción y la ayuda para que exista el aprendizaje. Considera también que tanto la lectura como la escritura son procesos de interpretación a través de los cuales se amplían y construyen los conocimientos del mundo exterior. Por otro lado, Montealegre y Forero (2006) considera que en la preparación del infante para llegar a la lectoescritura, influyen diferentes factores estos son: los factores intelectuales, los cuales están relacionados a la estimulación intelectual comprendiendo la inteligencia general y las habilidades del niño; los factores psicológicos, en los cuales consideran el afecto, autoestima, personalidad, madurez emocional; los factores sociales, referidos a la cultura y el ambiente y entorno factores orgánicos o fisiológicos refería la edad el sexo o las facultades que tiene el niño.

\section{MÉTODO}

El estudio es de tipo básico, ya que no tiene fines prácticos inmediatos y su objetivo es aumentar los conocimientos (Ñaupas et al., 2018). Es de bajo un diseño descriptivo de corte correlacional, ya que describe las características y datos de la población de estudio, además da a conocer la relación entre las variables de investigación analizando su vínculo. Ya que no se manipularon deliberadamente las variables y se basó principalmente en observar el fenómeno en el contexto natural para su posterior análisis (Hernández y Mendoza, 2018).

La población fue de 120 estudiantes de 5 años y la muestra estuvo conformada por 90 estudiantes. Se utilizó como instrumento el test TEPSI correspondiente a la variable desarrollo psicomotor, el cual comprende 52 ítems, los cuales estuvieron divididos en tres según sus dimensiones: motricidad, lenguaje y coordinación con 12,24 y 16 ítems respectivamente, este test permite conocer el rendimiento respecto a la evolución psicomotora de los niños de 2 a 5 años. Por otro lado, respecto a la variable iniciación a la lectoescritura se utilizó el test BENHALE, también conocido como batería evaluadora de habilidades necesarias para el aprendizaje de la lectura y escritura de Mora (1999), el cual estuvo conformado por 80 ítems. Ambos test fueron aplicados de forma individual a los alumnos de 5 años, el tiempo de administración de este instrumento fue de 30 a 40 minutos.

Los instrumentos fueron validados por juicio de expertos. Asimismo, para la confiabilidad, se realizó una prueba piloto con 30 niños y se utilizó el estadístico coeficiente 20 Kuder y Richardson, cuyos resultados demostraron una alta confiabilidad.

Para el análisis de datos, se realizó una prueba de normalidad para cada variable, a fin de determinar si los datos correspondían a una distribución normal; según el cálculo con el estadígrafo KolmogorovSmimov cuando $\mathrm{n} \geq 30$, se tuvo que los datos para la variable desarrollo psicomotor y aprendizaje a la lectoescritura no se aproximan a una distribución normal. Por lo que, por la naturaleza de las variables, se optó por utilizar el coeficiente de correlación de Spearman para medir la asociación o correlación entre las variables. 


\section{RESULTADOS Y DISCUSIÓN}

\section{Prueba de la Hipótesis general}

Respecto al desarrollo psicomotor y el aprendizaje a la iniciación de la lectoescritura, de acuerdo a los resultados obtenidos por el coeficiente de correlación de Spearman se tiene el p valor= $0,00<0,005$ y el coeficiente de rho de Spearman
$=0,599$ por lo que se confirma que existe una relación significativa positiva y moderada entre las variables desarrollo psicomotor y aprendizaje a la iniciación de la lectoescritura. Es así que se acepta la hipótesis alterna $\mathrm{H1}$ : Existe relación significativa entre el desarrollo psicomotor con el aprendizaje a la iniciación de la lectoescritura (Tabla 1).

Tabla 1. Correlación entre el desarrollo psicomotor y el aprendizaje a la iniciación de la lectoescritura.

\begin{tabular}{|c|c|c|c|c|}
\hline & & & Desarrollo Psicomotor & Aprendizaje Lectoescritura \\
\hline \multirow[t]{6}{*}{ Rho de Spearman } & \multirow{3}{*}{$\begin{array}{l}\text { Desarrollo } \\
\text { Psicomotor }\end{array}$} & Coeficiente de correlación & 1,000 &, $599^{* *}$ \\
\hline & & Sig. (bilateral) & . &, 000 \\
\hline & & $\mathrm{N}$ & 90 & 90 \\
\hline & \multirow{3}{*}{$\begin{array}{l}\text { Aprendizaje } \\
\text { Lectoescritura }\end{array}$} & Coeficiente de correlación &, $599^{\star *}$ & 1,000 \\
\hline & & Sig. (bilateral) &, 000 & . \\
\hline & & $\mathrm{N}$ & 90 & 90 \\
\hline
\end{tabular}

\section{Hipótesis Específica 1}

Respecto a la relación entre la dimensión lenguaje del desarrollo psicomotor y el aprendizaje de iniciación de la lectoescritura, de acuerdo a los resultados obtenidos por el coeficiente de correlación de Spearman se tiene el p valor $=0,00<$ 0,005 y el coeficiente de rho de Spearman $=0,460$ por lo que se afirma que existe una relación significativa positiva y moderada entre las variables lenguaje y aprendizaje a la iniciación de la lectoescritura. Por lo que se acepta la hipótesis alterna H1: Existe relación significativa entre el lenguaje con el aprendizaje a la iniciación de la lectoescritura (Tabla 2).

Tabla 2. Correlación entre el lenguaje y el aprendizaje a la iniciación de la lectoescritura.

\begin{tabular}{|c|c|c|c|c|}
\hline & & & Aprendizaje Lectoescritura & Lenguaje \\
\hline \multirow[t]{6}{*}{ Rho de Spearman } & \multirow{3}{*}{$\begin{array}{l}\text { Aprendizaje } \\
\text { Lectoescritura }\end{array}$} & Coeficiente de correlación & 1,000 &, $460^{\star *}$ \\
\hline & & Sig. (bilateral) & . & ,000 \\
\hline & & $\mathrm{N}$ & 90 & 90 \\
\hline & \multirow[t]{3}{*}{ Lenguaje } & Coeficiente de correlación &, $460^{\star *}$ & 1,000 \\
\hline & & Sig. (bilateral) &, 000 & . \\
\hline & & $\mathrm{N}$ & 90 & 90 \\
\hline
\end{tabular}

\footnotetext{
**. La correlación es significativa al nivel 0,01 (bilateral).
} 


\section{Hipótesis Específica 2}

Respecto a la relación entre la dimensión coordinación del desarrollo psicomotor y el aprendizaje de iniciación de la lectoescritura, según lo obtenido por el coeficiente de correlación de Spearman se tiene el $\mathrm{p}$ valor $=0,00<0,005 \mathrm{y}$ el coeficiente de Spearman $=0,546$ por lo que se afirma que existe una relación significativa positiva y moderada entre la coordinación y el aprendizaje a la iniciación de la lectoescritura. Por lo que se acepta la hipótesis alterna $\mathrm{H} 2$ : Existe relación significativa entre la coordinación con el aprendizaje a la iniciación de la lectoescritura (Tabla 3).

Tabla 2. Correlación entre el aprendizaje a la iniciación de la lectoescritura y la coordinación.

\begin{tabular}{lllrr}
\hline & & Aprendizaje Lectoescritura & Coordinación \\
\hline \multirow{2}{*}{ Rho de Spearman } & Aprendizaje & Coeficiente de correlación & 1,000 &, $546^{* *}$ \\
& Lectoescritura & Sig. (bilateral) & $\cdot$ &, 000 \\
& & N & 90 & 90 \\
& \multirow{2}{*}{ Coordinación } & Coeficiente de correlación &, $546^{* *}$ & 1,000 \\
& & Sig. (bilateral) &, 000 & $\cdot$ \\
& $\mathrm{N}$ & 90 & 90 \\
\hline
\end{tabular}

**. La correlación es significativa al nivel 0,01 (bilateral).

\section{Hipótesis Específica 3}

Respecto a la relación entre la dimensión motricidad del desarrollopsicomotoryel aprendizaje de iniciación de la lectoescritura, según lo obtenido por el coeficiente de correlación de Spearman se tiene el $\mathrm{p}$ valor $=0,00<0,005$ y el coeficiente de Spearman $=0,550$. Por lo que las variables tienen una correlación moderada. Aceptando la hipótesis alterna H3: Existe relación entre el aprendizaje a la iniciación de la lectoescritura y la motricidad. Es decir, si la motricidad incrementa, incrementarán los valores de aprendizaje a la iniciación a la lectoescritura. (Tabla 4).

Tabla 4. Correlación entre la motricidad y el aprendizaje a la iniciación de la lectoescritura.

\begin{tabular}{|c|c|c|c|c|}
\hline & & & Aprendizaje Lectoescritura & Motricidad \\
\hline \multirow[t]{6}{*}{ Rho de Spearman } & \multirow{3}{*}{$\begin{array}{l}\text { Aprendizaje } \\
\text { Lectoescritura }\end{array}$} & Coeficiente de correlación & 1,000 &, $550^{\star *}$ \\
\hline & & Sig. (bilateral) & . &, 000 \\
\hline & & $\mathrm{N}$ & 90 & 90 \\
\hline & \multirow[t]{3}{*}{ Motricidad } & Coeficiente de correlación &, $550^{* *}$ & 1,000 \\
\hline & & Sig. (bilateral) &, 000 & . \\
\hline & & $\mathrm{N}$ & 90 & 90 \\
\hline
\end{tabular}

\footnotetext{
**. La correlación es significativa al nivel 0,01 (bilateral).
} 


\section{Discusión}

Según los resultados obtenidos se coincide con los obtenidos por Pacori y Mamani (2020) quienes en su investigación determinaron la relación entre la lectoescritura en niños de 4 años de una institución educativa privada en Juliaca, Perú y su desarrollo psicomotor. Demostrando una relación significativa alta y positiva. Del mismo modo, Espinoza et al. (2017) determinaron la relación entre la adquisición de escritura en niños de una institución educativa pública de Perú y el desarrollo psicomotor, en donde destacaron que el desarrollo psicomotor y la adquisición de la escritura presentan una correlación directa. Asimismo, Saavedra y Tello (2015) obtuvieron resultados similares, en un grupo etario de niños de 5 años pertenecientes a una institución educativa Shipiba de Yarinacocha, en donde se rescata que si bien factores como el contexto pueden influir en el desarrollo psicomotor de los infantes, la relación significativa entre las variables ha resultado constante en investigaciones realizadas en diferentes áreas de Perú.

Por su parte Espósito et al., (2018) en su estudio sobre la elaboración de normas a nivel local para el test de desarrollo psicomotor en infantes argentinos, detallaron la importancia en la construcción de normas locales que presenten actualizaciones que tengan en cuenta características socioculturales a nivel local, para poder obtener resultados confiables y precisos. En ese sentido, Astudillo et al., (2018) en su investigación realizada en Chile, en su estudio respecto al desarrollo de los infantes de 0 a 4 años en el curso de la infancia indígena, indicaron que la naturaleza y la espiritualidad son factores importantes en el desarrollo de los infantes indígenas, por lo que valorar el desarrollo basándose solamente en el desarrollo psicomotriz resulta insuficiente para una valoración integral y compleja de las habilidades y destrezas de los niños indígenas. $\mathrm{Al}$ respecto, es importante tener en cuenta lo manifestado por Espósito et al. (2018) sobre que muchos de los factores que pueden influir en el desarrollo psicomotor del infante no están necesariamente incluidos en los test como el TEPSI, por lo que es necesario una contextualización de este test de acuerdo al entorno local en el que el niño se desarrolla. Del mismo modo, Sellés (2018) plantea la necesidad de un diseño de pruebas nuevas, que permitan evaluar de forma conjunta las habilidades que estén relación con el desarrollo inicial de la lectura y que estos test tengan la capacidad de detectar cuando los niños presentan alguna dificultad en la iniciación a la lectura.

Por otro lado, la investigación de Roberto (2018) fue utilizado como antecedente metodológico ya que estudió las dimensiones lenguaje, motricidad y coordinación en estudiantes de 5 años de la ciudad de Paraná, Argentina. Asimismo, utilizaron el test TEPSI. Del mismo modo, Leiva y Valdés (2016) en su estudio evaluaron a un grupo etario de 4 a 5 años en función a su nivel socioeconómico aplicando el test TEPSI. Respecto a la variable socioeconómica, se utilizó como instrumento una encuesta sociodemográfica, la cual fue dada a los padres de familia. Donde según los resultados, confirman una relación significativa entre el nivel educacional del jefe de familia con el desarrollo psicomotor. Los autores diseñaron tres modelos en los cuales permitieron una predicción en el desarrollo psicomotor en base al ingreso per cápita, el segundo modelo predijo el puntaje que se obtendría en la dimensión lenguaje en función 
al ingreso per cápita, finalmente el tercer modelo predijo el puntaje que se obtendría en cuanto a la coordinación en relación al nivel socioeconómico del jefe de familia.

De acuerdo con el desarrollo psicomotor y el aprendizaje de la lectoescritura, existe una relación significativa, según Doussoulin (2003) se desprende que al aprendizaje de la lectoescritura sugiere un desarrollo multidimensional, en el que se realizan cambios continuos y el infante se desenvuelve en niveles cada vez más complejos involucrando cambios en el plano físico o motor, intelectual y sensorial. Asimismo, Rodríguez et al. (2019) en su estudio realizado en Colombia, analizaron los proyectos de lectura y escritura en alumnos de instituciones públicas y privadas de Bucaramanga, destacando que existe una gran necesidad de fortalecer los proyectos de educación, reforzando los currículos escolares de manera que sean activos, lúdicos innovadores y creativos.

Por otro lado, es importante tener en cuenta lo descrito por Montealegre y Forero (2006) quien considera que en la preparación para que el infante llegue a la lectoescritura, influyen factores intelectuales, psicológicos, sociales y factores relacionados con el entorno. Esto permite señalar que los docentes tengan en cuenta todos los factores que podrían estar influenciando en la diferencia que tienen los niños en una misma aula respecto al aprendizaje de la lectoescritura. Asimismo, respecto al desarrollo psicomotor resaltan Sáez et al. (2021) quienes concluyen que existe una relación positiva entre el rendimiento académico y la habilidad psicomotora y además afirman que las habilidades lógico matemáticas y lingüísticas se benefician con la motricidad.

\section{CONCLUSIONES}

Se concluye que existe una relación significativa entre el desarrollo psicomotor y el aprendizaje de la iniciación a la lectoescritura en infantes de cinco años de una institución educativa de Lima. Por lo que si se implementa y profundiza en programas que favorezcan el desarrollo psicomotor del estudiante teniendo en cuenta su contexto, este tendrá una mejor predisposición respecto al aprendizaje de la lectoescritura.

Asimismo, existe una relación significativa entre las dimensiones lenguaje, coordinación y motricidad del desarrollo psicomotor y el aprendizaje a la iniciación de la lectoescritura en infantes de cinco años de una institución educativa de Lima. Por lo que es importante que los docentes implementen programas activos, creativos, lúdicos e innovadores que favorezcan el desarrollo psicomotor del estudiante y profundicen en las dimensiones lenguaje, coordinación y motricidad a fin que el estudiante tenga una mejor predisposición respecto a la lectoescritura.

\section{REFERENCIAS}

Arteaga, P., Dolz, V.. Droguett, E., Molina, P., Yentzen, G. (2001). Evaluación de Desarrollo Psicomotor en Lactantes y preescolares. Los Andes, Chile, 1999. Revista Chilena Salud Publica, 5 (1), 19-23.

Astudillo, P., Alarcón, A. M., Pérez, S., Fernández, F., Carmona, V., Castro, M., y Alarcón, S. (2018). Desarrollo psicomotor de 0 a 4 años en infancia indígena. Revisión sistemática de la literatura. Revista Chilena de Pediatría, 89(ahead), 0-0. https://doi.org/10.4067/s037041062018005000402

Diez de Ulzurum, A. (1999). La enseñanza de la lectura: enfoques psicolingüístico y sociocultural. Barcelona: Grao 
Doussoulin, A. (2003). Influencia del nivel socioeconómico y la estimulación ambiental en el desarrollo psicomotor en preescolares. Revista de Kinesiología. (70)

Espinoza, N., Hinton, V., y Mori, M. (2017). Correlación entre desarrollo psicomotor y Adquisición de la escritura en niños del tercer ciclo de la institución educativa $3701 \mathrm{Fe} y$ Alegría no 1. 1-26

Espósito, A. V. L., Korzeniowski, C. G., y Santini, M. (2018). Normas preliminares del Test de Desarrollo Psicomotor (TEPSI) para niños argentinos de 3 y 4 años. Liberabit. Revista Peruana de Psicología, 24(1), 9-27. https://doi. org/10.24265/liberabit.2018.v24n1.02

Hernández, Y. M. (2021). La psicoomtricidad fina y la iniciación de la escritura en los estudiantes de $1^{\circ}$ grado de educación primaria

Hernández-Sampieri, R. y Mendoza, C (2018). Metodología de la investigación. Las rutas cuantitativa, cualitativa y mixta, Ciudad de México, México: Editorial Mc Graw Hill Education, Año de edición: 2018, ISBN: 978-14562-6096-5, $714 \mathrm{p}$

Leiva, M., y Valdés, M. (2016). Modelo estadístico para predecir el puntaje de desarrollo psicomotor de niños de 4 a 5 años de edad en función del nivel socioeconómico: Proposal of a statistical model to predict the score of psychomotor development of children 4 to 5 years according to. Arch. Pediatr. Urug, 87(1), 22-27

Montealegre, R., y Forero, L. A. (2006). Desarrollo de la lectoescritura: adquisición y dominio. Acta Colombiana de Psicología, 9(1), 25-40

Mora, J. (1999) BEHNALE: Batería evaluadora de las habilidades necesarias para el aprendizaje de la lectura y escritura: manual. Madrid TEA D.L.

Ñaupas, H., Valdivia, Palacios y Romero (2018). Metodología de la investigación. Cualitativa, cuantitativa y redacción de tesis. $5^{\text {a }}$ Edición Grisley

OCDE (2017). Mejores políticas para una mejor vida. (s/f). Programa Internacional de Evaluación de los Alumnos (PISA). Recuperado de: http:// www.oecd.org/centrodemexico/medios/(30, 11, 2017)
Organización delas Naciones Unidas. (2017). Rendir cuentas en el ámbito de la educación: cumplir nuestros compromisos. http://acreditacion.unsl. edu.ar/wpcontent/ uploads/documentos $\% 20$ de\%20interes/educacion\%20superior/261016S. pdf

Pacori, F., y Mamani, L. (2020). Desarrollo psicomotor y madurez para la lecto escritura en niños de cuatro años de educación inicial Juliaca, 2020

Roberto, M. (2018). El desarrollo psicomotor (coordinación, lenguaje y motricidad) en niños de 5 años, de la ciudad de Paraná. Biblioteca Digital de La Universidad Católica Argentina, 18-25. http://bibliotecadigital.uca. edu.ar/repositorio/tesis/desarrollo-psicomotorcoordinacion-lenguaje.pdf

Rodríguez, N., Portilla, A., y Vera, A. (2019). La lectura y la escritura, una ventana al universo de los niños. Revista Del Instituto de Estudios En Educación y Del Instituto de Idiomas, Universidad Del Norte, 30, 63-81. https://doi. org/http://dx.doi.org/10.14482/zp.30.372.4 La

Rosso L. (2008). Psicología de la Educación para Padres y Profesionales ED. Radar Lescos Argentina

Saavedra, L., y Tello, P. (2015). Nivel de madurez para la lectoescritura en niños y niñas de 5 años de las instituciones educativas shipibas del nivel inicial del distrito de Yarinacocha - 2015

Sáez, M. B., Gil, P., y Martínez, M. (2021). Desarrollo psicomotor y su vinculación con la motivación hacia el aprendizaje y el rendimiento académico en Educación Infantil. Revista de Educacion. https://doi.org/10.4438/1988-592XRE-2021-392-483

Sellés, P. (2018). Estado actual de la evaluación de los predictores y de las habilidades relacionadas con el desarrollo inicial de la lectura. Pensamiento Educativo, 55(2), 53-71. https:// doi.org/10.7764/PEL.55.2.2018.9

Stone, R. (2019). Lectoescritura inicial en Latinoamérica y el Caribe: una revisión sistemática. Revista de Investigación $\mathrm{y}$ Evaluación Educativa, 6(1 ), 23-37 